\title{
On the Modern Clinical Application of Wendan Decoction
}

\author{
Yina Xiao', Mingjun Zhao' ${ }^{2}$, Zijuan He1, Gejing Chen', Mingming Wei ${ }^{1}$ \\ ${ }^{1}$ Shaanxi University of Chinese Medicine, Xianyang 712000, Shaanxi Province, China \\ ${ }^{2}$ Affiliated Hospital of Shaanxi University of Traditional Chinese Medicine, Xianyang 712000, Shaanxi Province, China \\ *Corresponding author: Mingjun Zhao, zmj7125@163.com
}

\begin{abstract}
Wendan Decoction is one of the commonly used prescriptions in clinical practice.it has a wide range of applications. On the basis of traditional Chinese medicine (TCM) syndrome differentiation, as long as it can be added or subdivided flexibly, it can be used to conquer various difficult and miscellaneous diseases seen in daily life.
\end{abstract}

Keywords: Wendan Decoction; Clinical application; The solution

Publication date: September 2021; Online publication: September 30, 2021

\section{Introduction}

Wendan Decoction comes from the important prescription for emergency preparation. It is composed of Rhizoma Pinelliae, Caulis Bambusae in Taenia, Fructus Aurantii Immaturus, dried orangepeel, Poria cocos fuling, and Radix Glycyrrhizae. It has the effects of regulating Qi-flowing for eliminating phlegm, clearing gallbladder and stomach ${ }^{[1]}$. Attending by the gallbladder depression phlegm disturbance caused by timidity easily startled, dizziness, palpitations, upset sleepless, and more different dreams in night; Or nausea, vomiting, hiccups, dizziness, epilepsy, whitish glossy coating of the tongue and slippery pulse string. The most common diseases treated are: Psychoneurosis, climacteric syndrome, schizophrenia, epilepsy, aural vertigo, bronchitis, coronary heart disease, peptic ulcer, chronic gastritis, cerebrovascular accident and other diseases ${ }^{[2]}$. At present, Wendan Decoction has been widely applied to the departments of internal medicine, otolaryngology, surgery, ophthalmology, pediatrics, infection and dermatology, etc., and also involves some difficult and complicated diseases, as well as still has a wide application prospect and development space ${ }^{[3]}$.

\section{Mental and nervous system diseases}

The etiology of vertigo syndrome in western medicine research is complex. Peripheral vertigo accounts for about $80 \%$. The most common is benign paroxysmal positional vertigo, followed by cervical vertigo, hypertension, arteriosclerosis, stroke and so on. Vertigo in traditional Chinese medicine is due to wind, fire, phlegm, deficiency and stasis, so there are "no dizziness without wind," "no dizziness without phlegm," "no dizziness without deficiency." Wendan Decoction is applicable to vertigo caused by phlegm turbidity and obstruction. Clinically, it is a kind of disease dominated by dizziness and visual rotation ${ }^{[4]}$. Shengli Yan ${ }^{[4]}$ and others randomly divided 80 patients with the disease into two groups and treated them with Wendan Decoction and Western Medicine (oral sibelium 10mg, if vomiting, then adds metoclopramide $10 \mathrm{mg}$ ). The total effective rate of Wendan Decoction group was 95\%, which was much higher than $80 \%$ of Western medicine group ( $\mathrm{p}<0.05$ ), and the cure course of Wendan Decoction was significantly shorter than that of Western medicine group. The study concluded that: Wendan Decoction is a famous prescription 
of traditional Chinese medicine and an important agent for treating phlegm. Clinically, Wendan Decoction has satisfactory effects on dizziness, nausea and stupidity, abdominal fullness and distention caused by phlegm heat and up to disturb as well as the resistance of phlegm turbidity. As long as it is diagnosed and treated according to syndrome differentiation and correctly added or subtracted drugs, all will get desirable satisfactory results.

\section{Cardiovascular diseases}

Hypertension and coronary heart disease are common diseases in cardiology. Qin Ouyang ${ }^{[5]}$ and others randomly divided 60 patients with coronary heart disease into observation group and control group. The control group was given isosorbide mononitrate tablet 40mg, and the observation group was given Wendan Decoction on the basis of the treatment of the control group. The degree of coronary stenosis before and after treatment was observed by coronary CTA: The coronary Gensini score of the patients was calculated, and the total effective rate of the observation group was $90.00 \%$, and that of the control group was $63.33 \%$ ( $\mathrm{p}<0.05$ ). The results showed that the treatment of angina pectoris caused by Qi-deficiency and phlegm obstruction coronary heart disease with Shiwei Wendan Decoction combined with western medicine was more effective than that with western medicine alone under the observation of coronary CTA. In addition, Wendan Decoction is also widely used in the treatment of other cardiovascular diseases, with obvious effect and high safety factor, which is worthy of clinical use.

\section{Respiratory diseases}

Chronic obstructive pulmonary disease (COPD) is one of the common diseases of the respiratory system. At present, the etiology of COPD is not fully clarified, so there is no radical cure means ${ }^{[6]}$. Tianhao $\mathrm{Li}^{\text {[7] }}$ and others randomly divided 42 patients with COPD in stable stage complicated with anxiety and depression into control group and treatment group, 22 cases in control group and 20 cases in treatment group. Two groups of patients were given Budesonide Formoterol (320 ug / 9 ug) with 2 times/day inhalation, and given anti-anxiety depression treatment. On the basis of the above treatment, the treatment group was treated with wendan Decoction for 4 weeks as a course of treatment. The results showed that Wendan Decoction combined with western medicine for the treatment of COPD combined with anxiety and depression, not only can significantly improve the lung function of patients, but also make patients anxiety and depression symptoms were significantly improved, and no obvious adverse reactions.

\section{Digestive diseases}

Bile reflux gastritis is one of the common diseases of digestive system. It is usually caused by excessive duodenal reflux in the non-surgical stomach. The duodenum, bile and other intestinal fluids mix with each other and flow back to the stomach of the body, which produces strong stimulation to the stomach of the body. Therefore, it is induced into bile reflux gastritis. Wei Zhao ${ }^{[8]}$ and others randomly divided 60 patients into two groups. 30 patients in the treatment group were given Wendan Decoction and 30 patients in the control group were given domperidone and famotidine capsules orally. The course of treatment in both groups was 4 weeks. Results: The effective rate of the treatment group was better than that of the control group. In a conclusion, Wendan Decoction has a significant clinical effect on bile reflux gastritis.

\section{Summary}

Wendan Decoction has achieved good results in the treatment of diabetes, phlegm dampness infertility and Facial Recurrent Dermatitis. It is widely used in clinical practice, and Chinese medicine prescriptions also have great potential for excavation. Our generation has the obligation and responsibility to carry out this heavy responsibility. 


\section{Disclosure statement}

The author declares no conflict of interest.

\section{References}

[1] Zhang XR, Liu XY, Xiang XX, 2010, Clinical Observation on 40 cases of Girls with Precocious Puberty Treated with Modified Wendan Decoction. Chinese Journal of Information on Traditional Chinese Medicine, 11: 79-80.

[2] Mao B, Wang G, Fan T, et al., 2007, Changes and Trends of Disease Spectrum in Randomized Controlled Trials of Traditional Chinese Medicine. Chinese Journal of Integrated Traditional and Western Medicine, 05: 404-408

[3] Xu HQ, Jia N, 2020, Clinical Application of Lonicera and Forsythia Powder. Journal of Liaoning University of Traditional Chinese Medicine, 02:164-167

[4] Yan SL, Yan HF, Gao L, 2019, Clinical Effect of Wendan Decoction on Vertigo Syndrome. World Latest Medicine Information, 61: $162+164$

[5] Ouyang Q, Ma Z, Zhou JH, 2020, Clinical Effect of Shiwei Wendan Decoction on Angina pectoris of Coronary Heart Disease by Coronary CTA Technique. Hubei Journal of Traditional Chinese Medicine, 05: 34-36.

[6] Zhou Z, 2014, Clinical Study on the Sequential Treatment of Kidney Deficiency and Phlegmdampness Polycystic Ovary Syndrome with Daying-35 and Tonifying Kidney Huatan Prescription. Zhejiang Chinese Medical University.

[7] Li TH, Ma JG, Gao XJ, et al., 2019, Evaluation of Clinical Efficacy of Wendan Decoction on COPD Combined with Anxiety and Depression. Modern Traditional Chinese Medicine, 06: 69-72.

[8] Zhao W, Yao C, 2008, Clinical Observation on the Treatment of Bile Reflux Gastritis by Wendan Decoction. Journal of Liaoning University of Traditional Chinese Medicine, 2008, 07: 98-99. 